\title{
Mind-muscle connection: effects of verbal instructions on muscle activity during bench press exercise
}

Antonio Paoli (1,2), Laura Mancin (3), Matteo Saoncella (4), Davide Grigoletto (1), Francesco Q. Pacelli (1), Paola Zamparo (5), Brad J. Schoenfeld (6), Giuseppe Marcolin (1)

(1) Department of Biomedical Sciences, University of Padua, Padua, Italy; (2) Research Center for High Performance Sport, UCAM, Catholic University of Murcia, Murcia, Spain; (3)Department of Human Sciences and Promotion of the quality of Life, San Raffaele, Roma, Italy; (4) Human Movement Sciences, University of Padova, Italy; (5) Department of Neuroscience, Biomedicine and Movement Sciences, University of Verona, Verona, Italy; (6) Department of Health Sciences, Program of Exercise Science, City University of New York, Lehman College, New York, USA

This article is distributed under the terms of the Creative Commons Attribution Noncommercial License (CC BY-NC 4.0) which permits any noncommercial use, distribution, and reproduction in any medium, provided the original author(s) and source are credited.

\begin{abstract}
Different attentional foci may modify muscle activation during exercises. Our aim was to determine if it is possible to selectively activate the pectoralis major or triceps brachii muscles according to specific verbal instructions provided during the bench press exercise. 13 resistancetrained males $(25.6 \pm 5.4 \mathrm{yrs}, 182.7 \pm 9.1 \mathrm{~cm}, 86.4 \pm 9.7 \mathrm{~kg})$ underwent an electromyographic signals acquisition of the sternocostal head, clavicular head of the pectoralis major, the anterior deltoid, and the long head of the triceps brachii (LT) during bench press exercise. Participants performed one non-instructed set (NIS) of 4 repetitions at 50\% 1-repetition maximum (1-RM) and one NIS of 4 repetitions at $80 \%$ 1-RM. Four additional sets of 4 repetitions at $50 \%$ and $80 \%$ 1-RM were randomly performed with verbal instructions to isolate the chest muscles (chest instructed set, CIS) or to isolate the triceps muscles (triceps instructed set, TIS). Participants showed significantly higher LT activation during TIS compared to non-instructed set both at $50 \%(\mathrm{p}=0.0199)$ and $80 \% 1-\mathrm{RM}(\mathrm{p}=0.0061)$ respectively. TIS elicited a significant $(\mathrm{p}=0.0250)$ higher activation of LT compared to CIS. Our results suggest that verbal instructions seem to be effective for increasing activity of the triceps brachii but not the pectoralis major during the bench press.
\end{abstract}

Key Words: resistance training; bench press; mind-muscle connection; internal focus.

Eur J Transl Myol 29 (2): 106-111, 2019

Attentional focus is considered an integral strategy in the field of motor learning. Attentional focus can be operationally defined as what an individual thinks about when carrying out a given movement or activity. ${ }^{1,2}$ There are two basic attentional focus strategies during performance of a task: internal and external. An internal focus involves thinking about a given bodily movement during performance while an external focus involves shifting performance-oriented concentration to the environment. There is compelling evidence that an external focus is superior to an internal focus for the execution of a wide array of tasks. A recent review of literature on the topic showed greater motor learning benefits when adopting an external focus in over $90 \%$ of published studies. ${ }^{3}$ These results held constant across a plethora of activities and outcome measures, thereby strengthening the rationale to focus externally when seeking to enhance physical performance. The application of attentional focus to resistance exercise remains somewhat equivocal. Limited research indicates that an external focus can enhance economy of movement during a lift by increasing force production and reducing ancillary muscular activity. ${ }^{4}$ It has been postulated that an internal focus may be more desirable to target specific muscles when the goal is to rehabilitate a musculoskeletal injury ${ }^{5}$ or promote regional-specific hypertrophy. ${ }^{6,7}$ Several studies have endeavoured to investigate the ability to selectively target different muscles, ${ }^{8-11}$ but the results have been conflicting. Moreover, there is evidence of an upper intensity 
Table 1. Demographics and resistance training variables $(n=13$, all men)

\begin{tabular}{ll}
\hline & Average \pm SD \\
\hline Age (years) & $25.6 \pm 5.4$ \\
Height $(\mathrm{cm})$ & $182.7 \pm 9.1$ \\
Body weight $(\mathrm{kg})$ & $86.4 \pm 9.7$ \\
RT experience (years) & $5.6 \pm 2.4$ \\
1-RM (kg) & $109.1 \pm 12.8$ \\
\hline
\end{tabular}

threshold whereby heavy load training diminishes the ability to activate a given muscle ${ }^{8}$ however, this findings requires confirmation. In these studies, surface electromyography (sEMG) was the most widely used method to quantify muscle activity while comparing different attentional foci during the bench press exercise. In one of the above-mentioned study, ${ }^{9}$ sEMG underlined that male football players could mainly increase the muscle activity of their bench press prime movers at $50 \%$ 1-repetition maximum (RM) compared to $80 \%$ 1-RM, after verbal instructions of focusing on those muscles. More recently, Calatayud ${ }^{8}$ pointed out that male recreationally males were able to modify triceps brachii and pectoralis major to a greater extent only for loads below 60\% 1-RM during bench press. On this topic and with a similar approach Daniels ${ }^{11}$ did not find significant differences in the sEMG activity of the bench press prime movers comparing trained and untrained males. Contrary to the author's hypothesis, trained males did not exhibit higher sEMG activity of the pectoralis and triceps muscles compared than the untrained males after verbal instructions to focus on chest or arm muscles.

The purpose of the present study was twofold: 1) To determine whether resistance-trained individuals could selectively target the triceps and pectorals during the barbell bench press by employing an internal, ${ }^{12}$ versus a control where no instructions were provided, and; 2) To determine if differences in activation exist when carrying out performance at 50\% 1RM versus $80 \% 1 \mathrm{RM}$.

\section{Materials and Methods}

\section{Participants}

Thirteen resistance trained males participated in this study (see Table 1). To be recruited, each participant was required to have at least 2 years of resistance training (RT) experience, performing at least 3 sessions per week at moderate to high intensity. In addition, they were familiarized with the barbell bench press exercise. Barbell bench press exercise is a multi-joint exercise that involves multiple muscle groups. ${ }^{13}$ Moreover, participants had not to present any injuries at shoulders, elbows, wrist and back over the last 6 months. All participants were informed about the purpose and content of the investigation. Informed consent was obtained from all individual participants included in the study. The experimental protocol was approved by the local Institutional Review Board (approval n. HECDSB12/16).

\section{Experimental procedures}

The general procedure for the study is outlined in Figure 1. Participants reported to the laboratory on one occasion. After an individual 10-minute warm up, they were asked to perform a 5-RM bench press test which allowed to estimate the 1-RM bench press. ${ }^{14-15}$ Then participants rested for an hour before being prepared for the sEMG data collection of the following muscles: sternocostal head (SP) and clavicular head (CP) of pectoralis major, anterior deltoid (AD), and long head of triceps (LT). To help control for the undue influence of external factors possibly affecting bench press performance, all measurements were conducted by the same investigator and in the same facility. Participants started all the bench press sets with fully extended arms and with a selfselected pronate grip width. Feet were kept at about shoulders width. During the eccentric phase, elbows flexed until the bar touched the chest. Scapula and spine were respectively hold retracted and in flat position.

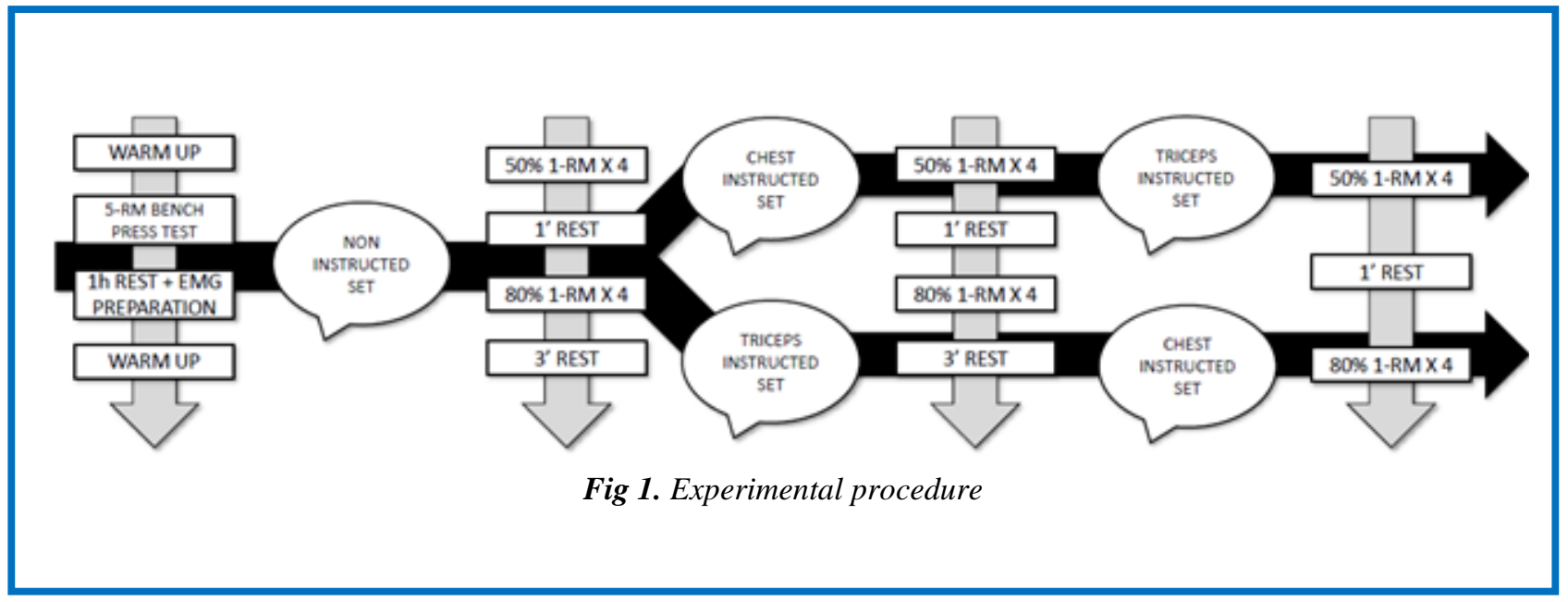


Participants were instructed to perform each repetition within a standardized time consisting of 2 seconds down, 1 second pause at the chest, and 1 second up. Time was manually controlled with a stopwatch by the investigator. Before the beginning of the experimental protocol, participants performed a second 5-minute individual warm up to familiarize with the worn sEMG instrumentation. After the warm up, the participants were instructed to perform 1 set of 4 repetitions of the bench press at $50 \%$ of $1-\mathrm{RM}$, which is often selected in RT programs Upper body muscle activation during lowversus high-load resistance exercise in the bench press, 15 and 1 set of 4 repetitions at $80 \%$ of $1-\mathrm{RM}$, which has been demonstrated to be correlated with an increase of the pushing muscles activity. ${ }^{16,17}$ After 3-minute rest period, participants performed 2 sets of 4 repetitions at $50 \%$ and $80 \%$ 1-RM respectively (separated by 1 minute). Verbal instructions were given before the beginning of the set to isolate chest muscles ("During this set, try to use only your chest muscles. To do this, attempt to bring your elbows to each other when you push.'"). After another 3-minute rest period, participants were asked to focus on the triceps muscles to complete the lift (triceps instructed set, TIS). This time the verbal instruction reported: "During this set, try to use only your triceps muscles. To do this, attempt to turn away your elbows from each other when you push.' The last two conditions with the verbal instructions were performed in a randomized order.

\section{Data collection analysis}

sEMG raw signals were recorded by means of a PDA PocketEMG (BTS Bioengineering, IT). To determine the lift cycle defined by two consecutive elbow extensions an electrogoniometer (Biometrics LTD, UK) was placed on the lateral side of the right elbow to measure its flexextension. Data were synchronously recorded at a sampling frequency of $1 \mathrm{kHz}$. Electrode placement followed the recommendations of the Surface Electromyography for Non-Invasive Assessment of Muscles (SENIAM) project. ${ }^{18}$ The skin surface over the centre of the muscle belly was shaved with a razor and cleaned by rubbing with an alcohol pad. Pre-gelled bipolar surface electrodes (Arbo, H124SG, Kendall) were placed with an interelectrode distance of $24 \mathrm{~mm}$. A single reference electrode was placed over the styloid process of the radius. Once the preparation was complete, signal quality was checked asking the participant to contract each muscle against a resistance. Raw sEMG signals obtained during the trials were rectified around their mean value, then integrated with a moving window of $150 \mathrm{~ms}$ and finally smoothed with a 4th order Butterworth low pass filter set at $5 \mathrm{~Hz}$. As reported in our previous studies ${ }^{19,20}$, the analysis of sEMG data was based on every set excluding the first and the last repetition. For each set, the mean value of the two repetitions considered was calculated. Than the mean of the means was reported for each muscle. sEMG data analysis was performed by the Smart Analyzer software (BTS Bioengeneering, Milano, Italy).

\section{Statistical Analysis}

A two-way repeated measure ANOVA (2 load intensities $\mathrm{x} 3$ verbal instructions) was used to establish whether there were significant main effects and/or interactions between the two independent variables. When a significant main effect or interaction $(\mathrm{P}<0.05)$ was found, a Bonferroni post-hoc pairwise comparison was conducted to determine the origin of these differences. All statistical analyses were computed by means of the

\begin{tabular}{|c|c|c|c|c|}
\hline \multicolumn{5}{|c|}{ Table 2. sEMG activity at 50\% and $80 \% 1-R M$ (mean and SD). } \\
\hline & & NIS & CIS & TIS \\
\hline \multirow{2}{*}{ SP } & $50 \%$ & $0.206 \pm 0.106$ & $0.228 \pm 0.110$ & $0.187 \pm 0.097$ \\
\hline & $80 \%$ & $0.326 \pm 0.126$ & $0.350 \pm 0.130$ & $0.394 \pm 0.177$ \\
\hline \multirow{2}{*}{$\mathbf{C P}$} & $\mathbf{5 0 \%}$ & $0.286 \pm 0.178$ & $0.382 \pm 204$ & $0.298 \pm 0.171$ \\
\hline & $\mathbf{8 0 \%}$ & $0.493 \pm 0.240$ & $0.584 \pm 0.263$ & $0.646 \pm 0.332$ \\
\hline \multirow{2}{*}{ LT } & $\mathbf{5 0 \%}$ & $0.134 \pm 100$ & $0.169 \pm 0.123$ & $0.196 \pm 0.163^{*}$ \\
\hline & $\mathbf{8 0 \%}$ & $0.294 \pm 0.208$ & $0.327 \pm 0.217$ & $0.364 \pm 0.215^{* *}$ \\
\hline \multirow{2}{*}{ AD } & $\mathbf{5 0 \%}$ & $0.277 \pm 0.189$ & $0.303 \pm 0.194$ & $0.288 \pm 0.179$ \\
\hline & $\mathbf{8 0 \%}$ & $0.477 \pm 0.309$ & $0.500 \pm 0.200$ & $0.500 \pm 0315$ \\
\hline
\end{tabular}

SP: sternocostal head of pectoralis major, CP: clavicular head of pectoralis major, LT: long head of triceps, AD: anterior deltoid, NIS: non-instructed set, CIS: chest instructed set, TIS: triceps instructed set. $(*=p<0.05$ NIS vs TIS; ** $=p<0.01$ NIS vs TIS) 


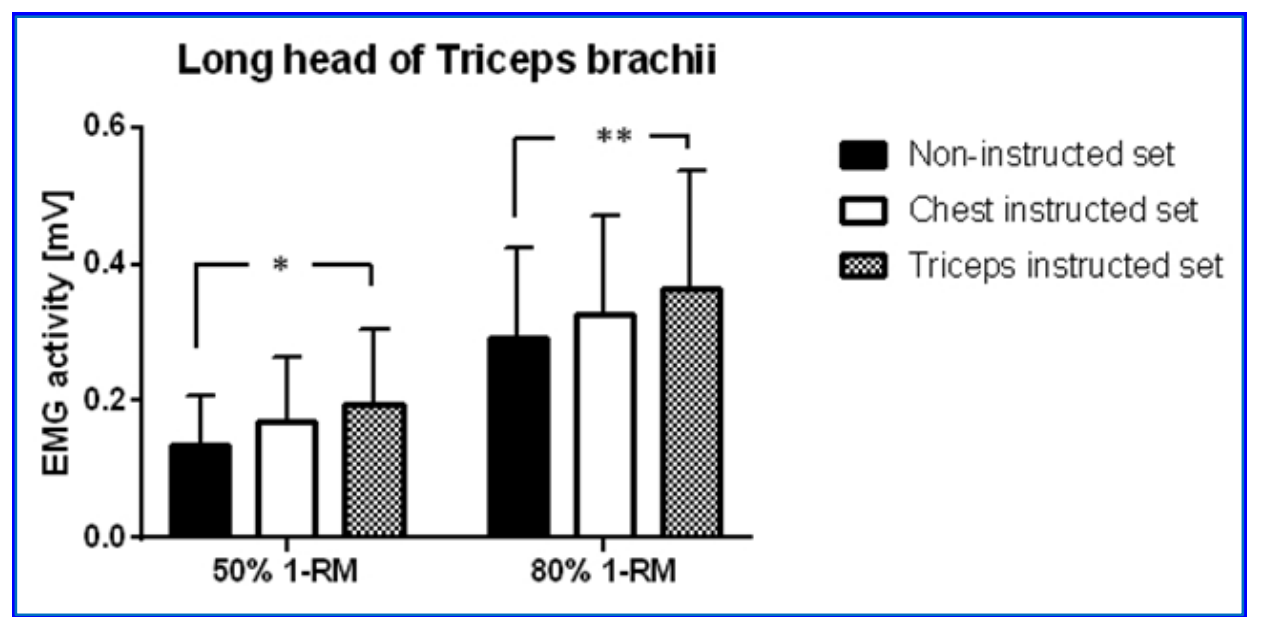

Fig 2. sEMG activity at 50\% and 80\% 1-RM (mean and SD). Data referred to LT (long Head of Triceps)

software package GraphPad Prism version 7.00 for Windows (GraphPad Software, San Diego California USA).

\section{Results}

Data of the sEMG activity of each muscle are presented in Table 2. A significant main effect of the load intensity was detected for all muscles: SP $(\mathrm{P}<0.001)$, CP $(\mathrm{P}<$ 0.01), LT ( $\mathrm{P}<0.001)$, AD $(\mathrm{P}<0.001)$. On the contrary, a significant main effect of verbal instructions was effective only for the LT $(\mathrm{P}<0.01)$ (Figure 2$)$. The Bonferroni post hoc test underlined differences between $50 \%$ non-instructed set (NIS) vs 50\% TIS $(\mathrm{P}<0.05)$ and $80 \%$ NIS vs $80 \%$ TIS $(\mathrm{P}<0.01)$. The other muscles were not affected by the verbal instructions $(\mathrm{SP}, \mathrm{p}=0.572 ; \mathrm{CP}$, $\mathrm{p}=0.165 ; \mathrm{AD}, \mathrm{p}=0.546)$. None interaction was found between the two independent variables.

\section{Discussion}

The present study showed that resistance-trained males can alter the activation of the LT, but not the pectorals, during both moderate and higher intensity bench press in response to verbal instruction. Previous research indicates that football players performing a bench press at 50\% 1-RM were able to increase both SP and LT activity while maintaining proper form and similar movement speed by shifting attentional focus to the respective muscles (by $22 \%$ and $25.7 \%$, respectively). ${ }^{9}$ However, during training at $80 \% 1-\mathrm{RM}$, only the PM showed increased activation from an internal focus while activity of the LT was statistically unchanged. More recently, Calatayud et al. ${ }^{8}$ found that activity of both the PM and LT can be increased by focusing on using the respective muscles at relative loads between 20 and 60 $\%$, but not at $80 \%$ of $1 \mathrm{RM}$. In contrast, results of our study show that at both $50 \%$ and $80 \% 1-\mathrm{RM}$, the subjects were capable of altering muscle participation of the elbow extensors, but not the horizontal adductors. Even though we used resistance trained males, the selective activation of muscles didn't occur because of the necessary effort needed to lift heavy weight by the prime movers. It seems that the subjects are primarily focused on lifting the weight when heavy weights are used. Using body weight as resistance, ${ }^{21,22}$ or loads, varying from 30 to $50 \%$ of the $1-\mathrm{RM},{ }^{10,23}$ produces greater muscle activation after specific verbal instructions in dynamic exercises as the most studies present in literature on the topic report. Unlike the study of Calatayud, ${ }^{8}$ the activation of the pectoralis major increased to a greater extent when subjects were asked to focus on the triceps at high intensity ( $80 \%$ of $1-\mathrm{RM})$. Moreover, even when subjects were asked to focus on the pectoral muscles, triceps activity showed an increase compared to the condition without focus. Our findings are in contrast with some previous research, where the activation of the alternate muscle (i.e triceps activity during CIS) as shown in our study was not observed.22,24 As proposed by Karst and Willet, ${ }^{22}$ subjects performing the abdominal crunch accompanied by verbal instructions could voluntary modify the activity of the rectus abdominis and external oblique in a univocal way. In fact, subjects were able to increase external oblique activity and to decrease rectus abdominis activity, but they were not able to do the opposite. Alternatively, subjects in our study were instructed to focus their attention on increasing the activity of a specific muscles and were not instructed to focus on the relaxation of the alternate muscle. This difference in instructions can illustrate the failure of "muscle isolation" in our study. It is also possible that the differences in muscles investigated between our study (pectorals and elbow flexors) and the others (abdominals and shoulder musculature, respectively $)^{22,24}$ may have at least in part accounted for the conflicting findings. A reasonable interpretation for greater muscle activity followed by specific verbal instructions could be found 
in the "constrained action hypothesis." This theory, described by Wulf et al. ${ }^{25}$ and supported by subsequent studies, ${ }^{4,26,27}$ explains the relative benefits of adopting an external rather than an internal focus of attention. According to this theory the body's automatic control of movements is made less functional when an internal attentional focus is used. In the review of this topic by Wulf $^{3}$, studies showing improved task-oriented performance with an external focus were matched with decreased sEMG of the working musculature. The evidence of an increase in sEMG activity with verbal instructions agrees with the findings of reported studies, although our study used a multi-joint exercise and included a control condition without any instructions. Our results confirm that an internal focus could be more useful when we want to increase the activity of a specific muscle; whereas an external focus could be more indicated when we want to achieve a performance improvement. Some questions still remain opened: it is unknown how the increased activity of a muscle could occur without modifying other aspects (e.g. kinematics) of movement. In our study, subjects did not succeed in reducing the LT activity after the CIS. However, we hypothesize that subjects in previous studies performed bench press keeping their arms very close to their body, which may have increased the activity of the $\mathrm{AD}, \mathrm{CP}$ and LT instead of reducing SP involvement. It is possible that fatigue may have been a confounding factor even though we randomized the different instruction sets order. However, excessive effort or fatigue were not reported by the subjects during exercises both at $50 \%$ and at $80 \% 1$ RM, making such speculation unlikely. Our study presents both strengths and limitations. Compared to other studies that investigated verbal instructions applied to a RT protocol, a strength of our study was the expertise in RT of our subjects. A limitation lies in not having measured the activity of the antagonist muscles such as reported in the study of Snyder and Fry. ${ }^{9}$ Finally, the amplitude of the sEMG signal reflects a combination of motor unit recruitment, firing rates and the degree of motor unit synchronization. ${ }^{28}$ Thus, it remains to be determined which of these factors, or combinations of them, ${ }^{29,30}$ generated the increase of the sEMG signal.

In conclusion, verbal instructions seem to be effective for increasing activity of the triceps brachii but not the pectoralis major during the bench press in resistance trained men. Future studies should investigate whether or not these effects could be increased through training with verbal instructions.

\section{List of acronyms}

CIS - chest instructed set

$\mathrm{CP}$ - clavicular head of the pectoralis major

LT - the long head of the triceps brachii

NIS - non-instructed set

$\mathrm{RM}$ - repetition maximum

RT- resistance training

sEMG - surface EMG
SP - sternocostal head of the pectoralis major TIS - triceps instructed set

\section{Authors contributions}

AP, PZ, GM, and FQP conceived the study; MS and DG performed the experiment; MS, GM and AP analysed the data; MS and AP wrote the paper; BS and LM helped to editing the manuscript.

\section{Acknowledgments None.}

Funding No funding was obtained for this research project.

\section{Conflict of Interest}

Authors declare no conflicts of interest.

\section{Ethical Publication Statement}

We confirm that we have read the Journal's position on issues involved in ethical publication and affirm that this report is consistent with those guidelines.

\section{Corresponding Author}

Antonio Paoli, Department of Biomedical Sciences, University of Padova, Via Marzolo, 335131 Padova (Italy), Ph. +390498275318, Fax +390498275301;

Email: antonio.paoli@unipd.it

E-mails of co-authors

Laura Mancin: mancin.laura.1@gmail.com Matteo Saoncella: matteo.saoncella@gmail.com Davide Grigoletto: davide.grigoletto@unipd.it Francesco Q. Pacelli: francesco.pacelli@unipd.it Paola Zamparo: paola.zamparo@univr.it

Brad J. Schoenfeld: bradschoenfeldphd@gmail.com Giuseppe Marcolin: giuseppe.marcolin@unipd.it

\section{References}

1. Schoenfeld BJ, Contreras B. Attentional Focus for Maximizing Muscle Development: The MindMuscle Connection. Strength \& Conditioning Journal 2016;38:27-9.

2. Schoenfeld BJ, Vigotsky A, Contreras B, Golden S, Alto A, Larson R, Winkelman N, Paoli A. Differential effects of attentional focus strategies during long-term resistance training. Eur J Sport Sci 2018;18:705-12. e-pub ahead of print 2018/03/14.

3. Wulf G. Attentional focus and motor learning: a review of 15 years. International Review of Sport and Exercise Psychology 2013;6:77-104.

4. Marchant DC, Greig M, Scott C. Attentional focusing instructions influence force production and muscular activity during isokinetic elbow flexions. J Strength Cond Res 2009;23:2358-66.

5. Andersen LL, Magnusson SP, Nielsen M, et al. Neuromuscular activation in conventional therapeutic exercises and heavy resistance exercises: implications for rehabilitation. Phys Ther 2006;86:683-97. e-pub ahead of print 2006/05/03. 
6. Wakahara T, Miyamoto N, Sugisaki N, Murata K, Kanehisa H, Kawakami Y, Fukunaga T, Yanai T. Association between regional differences in muscle activation in one session of resistance exercise and in muscle hypertrophy after resistance training. Eur J Appl Physiol 2012;112:1569-76. e-pub ahead of print 2011/08/23.

7. Wakahara T, Fukutani A, Kawakami Y, Yanai T. Nonuniform muscle hypertrophy: its relation to muscle activation in training session. Med Sci Sports Exerc 2013;45:2158-65. e-pub ahead of print 2013/05/10.

8. Calatayud J, Vinstrup J, Jakobsen MD, et al. Importance of mind-muscle connection during progressive resistance training. Eur J Appl Physiol 2016;116:527-33.

9. Snyder BJ, Fry WR. Effect of verbal instruction on muscle activity during the bench press exercise. J Strength Cond Res 2012;26:2394-400.

10. Snyder BJ, Leech JR. Voluntary increase in latissimus dorsi muscle activity during the lat pulldown following expert instruction. J Strength Cond Res 2009;23:2204-9.

11. Daniels RJ, Cook SB. Effect of instructions on EMG during the bench press in trained and untrained males. Hum Mov Sci 2017;55:182-8. epub ahead of print 2017/08/26.

12. Graupe D. Recognition and prediction of individual and combined muscular activation modes via surface EMG analysis. Eur J Transl Myol 2010;20:131-8.

13. Gentil P, Campos MH, Soares S, et al. Comparison of elbow flexor isokinetic peak torque and fatigue index between men and women of different training level. Eur J Transl Myol 2017;27:7070. e-pub ahead of print 2018/01/05.

14. Brzycki M. Strength testing-predicting a one-rep max from reps-to-fatigue. JOPERD 1993;64:88-90.

15. Schoenfeld BJ, Contreras B, Vigotsky AD, et al. Upper body muscle activation during low-versus high-load resistance exercise in the bench press. Isokinet Exerc Sci 2016;24:217-24.

16. Pinto R, Cadore E, Correa C, et al. Relationship between workload and neuromuscular activity in the bench press exercise. Medicina Sportiva 2013;17:1-6.

17. McCaw ST, Friday JJ. A comparison of muscle activity between a free weight and machine bench press. J Strength Cond Res 1994;8:259-64.

18. Hermens HJ, Freriks B, Disselhorst-Klug C, Rau G. Development of recommendations for SEMG sensors and sensor placement procedures. J Electromyogr Kinesiol 2000;10:361-74.

19. Marcolin G, Petrone N, Moro T, et al. Selective Activation of Shoulder, Trunk, and Arm Muscles: A Comparative Analysis of Different Push-Up Variants. J Athl Train 2015;50:1126-32.

20. Paoli A, Marcolin G, Petrone N. Influence of different ranges of motion on selective recruitment of shoulder muscles in the sitting military press: an electromyographic study. J Strength Cond Res 2010;24:1578-83.

21. Critchley D. Instructing pelvic floor contraction facilitates transversus abdominis thickness increase during low-abdominal hollowing. Physiother Res Int 2002; 7:65-75.

22. Karst GM, Willett GM. Effects of specific exercise instructions on abdominal muscle activity during trunk curl exercises. J Orthop Sports Phys Ther 2004;34:4-12.

23. Bressel E, Willardson JM, Thompson B, Fontana FE. Effect of instruction, surface stability, and load intensity on trunk muscle activity. J Electromyogr Kinesiol 2009;19:e500-4.

24. Palmerud G, Kadefors R, Sporrong H, et al. Voluntary redistribution of muscle activity in human shoulder muscles. Ergonomics 1995;38:806-15.

25. Wulf G, Prinz W. Directing attention to movement effects enhances learning: a review. Psychon Bull Rev 2001;8:648-60.

26. Vance J, Wulf G, Tollner T, et al. EMG activity as a function of the performer's focus of attention. $J$ Mot Behav 2004;36:450-9.

27. Wulf G, Dufek JS, Lozano L, Pettigrew C. Increased jump height and reduced EMG activity with an external focus. Hum Mov Sci 2010;29:4408.

28. Aagaard P. Training-induced changes in neural function. Exerc Sport Sci Rev 2003;31:61-7.

29. Vigotsky AD, Beardsley C, Contreras B, et al. Greater electromyographic responses do not imply greater motor unit recruitment and 'hypertrophic potential' cannot be inferred. J Strength Cond Res 2017;31(1):e1-e4. Epub 2015 Dec 11.

30. Vigotsky AD, Ogborn D, Phillips SM. Motor unit recruitment cannot be inferred from surface EMG amplitude and basic reporting standards must be adhered to. Eur J Appl Physiol 2016;116:657-8.

Submissions: April, 24, 2019

Acceptance: May, 06, 2019 